\title{
AVALIAÇÃO DE LFC'S NOS LIMITES DE TENSÃO DO PRODIST
}

\author{
Márcio Zamboti Fortes ${ }^{1}$ \\ Antônio Marcos Estrela Pereira ${ }^{2}$ \\ Adriano Pinheiros Fragoso ${ }^{3}$ \\ Geraldo Martins Tavares ${ }^{4}$
}

\begin{abstract}
Resumo: Existem no mercado brasileiro de equipamentos de iluminação, lâmpadas fluorescentes compactas (LFC) de diversos fabricantes que atendem a normatização específica de qualidade do Programa Brasileiro de Etiquetagem (PBE) que é acompanhada pelo Instituto Nacional de Metrologia, Qualidade e Tecnologia (INMETRO) através dos laboratórios certificadores. Estas lâmpadas são ensaiadas em condições nominais de tensão, o que muitas vezes não retrata a realidade no ponto final de conexão, nas instalações do consumidor. Este artigo apresenta uma avaliação da LFC dentro de condições limites de tensões regulamentadas pelos Procedimentos de Distribuição de Energia Elétrica no Sistema Elétrico Nacional - PRODIST, mostrando o comportamento de 10 amostras de fabricantes diferentes aprovados nos testes de requisitos para aceitação no mercado brasileiro. As lâmpadas foram ensaiadas frente às condições reais encontradas no usuário final, fazendo-se uma comparação dos resultados entre as amostras.
\end{abstract}

Palavras-Chave: Lâmpadas Fluorescentes Compactas; Qualidade da Energia; Luminotécnica.

\begin{abstract}
There are in the Brazilian lighting equipment market, compact fluorescent lamps (CFL) from different manufacturers that meet the quality standards according to Brazilian Labeling Program (PBE) which is accompanied by the National Institute of Metrology, Quality and Technology (INMETRO) through the certifying laboratories. These lamps are tested at nominal voltage conditions, which most of time does not demonstrate the reality at the end point of connection, at the user. This paper presents a CFL evaluation front voltage limits conditions regulated by electricity Distribution Procedures in Brazilian Electrical System - PRODIST. The paper demonstrates the behavior of 10 different manufacturers samples approved in standard tests for acceptance in the Brazilian market. The samples were tested against the real conditions found at the end user and the conclusion presents a comparison of the results.
\end{abstract}

Keywords: Compact Fluorescent Lamps; Energy Quality; Lighting.

\footnotetext{
${ }^{1}$ Departamento de Engenharia Elétrica da Universidade Federal Fluminense (UFF). E-mail: mzf@ vm.uff.br

${ }^{2}$ LABLUX (UFF). E-mail: am_estrela@id.uff.br

${ }^{3}$ Programa de Mestrado Profissional em Metrologia e Qualidade (INMETRO). E-mail: adrianofragoso@gmail.com

${ }^{4}$ Departamento de Engenharia Elétrica da Universidade Federal Fluminense (UFF). E-mail: gtavares@vm.uff.br
} 


\section{INTRODUÇÃO}

O governo brasileiro, em 1985, por meio dos Ministérios de Minas e Energia e de Indústria e Comércio, criou o Programa Nacional de Conservação de Energia Elétrica (PROCEL). O objetivo principal do PROCEL é "promover a racionalização da produção e do consumo de energia elétrica, para que se eliminem os desperdícios e se reduzam os custos e os investimentos setoriais" (PROCEL, 2013).

Devido à crise que ocorreu no setor elétrico brasileiro em 2001, foi publicada a lei 10.295 de 17 de outubro de 2001 (PRESIDÊNCIA DA REPÚBLICA, 2013) que estabelecia uma política nacional de eficiência energética voltada para máquinas e aparelhos consumidores de energia no Brasil. Logo em seguida foi publicado o decreto 4.059 de 19 de dezembro de 2001 (PRESIDÊNCIA DA REPÚBLICA ${ }^{2}$, 2013). Por meio deste, ficou regulamentada a lei que define os pontos a serem avaliados em cada produto e designado o INMETRO como o órgão responsável pelos programas de fiscalização e avaliação da conformidade de tais produtos. A partir de então, cresceu a demanda por laboratórios para realização de testes nas diversas categorias de dispositivos e equipamentos elétricos.

Em 2005, o INMETRO concedeu à Universidade Federal Fluminense (UFF) o credenciamento para participar do Programa Brasileiro de Etiquetagem (PBE) por meio de seu Laboratório de Luminotécnica (LABLUX), em testes sobre lâmpadas fluorescentes compactas (LFC) com reator integrado, para comprovar os índices mínimos de eficiência energética, estabelecidos pelo regulamento de avaliação de conformidade de LFC's.

Quando busca-se na literatura trabalhos relacionados a ensaios e avaliação de características de LFC's, encontram-se trabalhos como o de Dilai e outros (2005) que avalia as características de frequência, eficiência luminosa e relação volt-ampere em dois diferentes modelos de LFC's e sinalizam a faixa de frequência ideal para funcionamento das amostras em teste. Outros estudos encontrados relatam sobre avaliação por simulação do comportamento das LFC's frente a variações de tensão e frequência, usando-se o circuito de um "dimmer" como exemplo, como o apresentado em (Shafi e McMahon, 2006).

Um trabalho com análises iniciais na mesma linha do desenvolvido neste artigo é apresentado por May e Collins (2010), onde foram ensaiadas duas lâmpadas incandescentes e três LFC's, comparandose o desempenho da intensidade luminosa em relação à tensão de saída, assim como, a resposta a subtensões com ciclos definidos.

Existem pesquisas mais especificas, que são importantes para o conhecimento do impacto real que tecnologias envolvidas com este tipo de lâmpadas trazem aos sistemas elétricos. Podem-se citar estudos focando alguns efeitos que as LFC's podem causar às redes de distribuição (Watson et. al., 2009), interferência de rádio frequência emitida sobre equipamentos (Schinkelshoek et. al., 2010) e interferência eletromagnética (Coca et. al., 2011).

Pesquisas relacionadas à qualidade das lâmpadas encontradas no mercado também são importantes para balizar as ações de certificação nos diversos países. $\mathrm{O}$ modelo de certificação adotado no Brasil é atual e está em frequente revisão dos procedimentos, limites e desempenho dos equipamentos ensaiados. Neste sentido, pode-se citar em especial o trabalho de Blanco et. al. (2011) que compara a qualidade das LFC's comercializadas na Colômbia e Alemanha. É um trabalho que apresenta a diferença entre os produtos comercializados nestes países e incentiva ainda mais ao conhecimento, testes, documentação e divulgação dos resultados dos produtos de outros mercados.

No presente trabalho analisam-se os resultados de desempenho de 10 amostras de fabricantes diferentes de LFC's disponíveis no mercado brasileiro. Os nomes dos fabricantes não serão divulgados por questões de confidencialidade que garantem a não exposição dos mesmos. As amostras ensaiadas foram coletadas no mercado de varejo da região sudeste do Brasil, e de fabricantes conhecidos nacionalmente e que já possuem autorização de comercialização para o modelo escolhido. No experimento realizado, buscou-se avaliar $\mathrm{O}$ 
comportamento destas amostras dentro da faixa limite de tensão aceitável no consumidor final segundo o PRODIST, com os valores limites estabelecidos entre $+5 \%$ e $-10 \%$. As amostras são estudadas com relação à corrente consumida, fator de potência, potência média consumida, eficiência energética e fluxo luminoso. São apresentados como indicadores, suporte para este estudo, três novas relações entre grandezas que apoiam as conclusões.

\section{DESCRIÇÃO DOS ENSAIOS PADRONIZADOS}

Os ensaios de lâmpadas fluorescentes compactas com reator integrado são regidos pela portaria INMETRO 489/2010 (2010). Inicialmente, 20 amostras ficam 100 horas energizadas de forma ininterruptas. Dentre as 20 amostras escolhem-se 10 unidades para realizarem-se os ensaios de: medição de potência elétrica, fator de potência, corrente elétrica, distorção harmônica total de corrente, fluxo luminoso, cálculo da eficiência luminosa, temperatura de cor, equivalência de fluxo luminoso médio para enquadramento dos produtos ao fluxo luminoso da lâmpada incandescente equivalente e determinação da classe de eficiência energética.

Após o ensaio de 100 horas e se forem alcançados os requisitos mínimos de eficiência luminosa exigidos em (INMETRO, 2010), as amostras passam para o ensaio de 2.000 horas, onde permanecem energizadas por 2 horas e 45 minutos e desenergizadas por 15 minutos, em cada ciclo de 3 horas, sendo admitido ao fim do ensaio no máximo $10 \%$ de queima e manutenção da eficiência luminosa de pelo menos $80 \%$ em relação ao valor medido na amostra sob teste de 100 horas.

Após o ensaio de 2.000 horas, iniciam-se os ensaios de vida mediana nominal, que é determinada pela média da queima entre a décima e décima primeira lâmpada.

Os ensaios elétricos e fotométricos são realizados com temperatura controlada de $25^{\circ} \mathrm{C} \pm 1{ }^{\circ} \mathrm{C}$, com tensão controlada, tolerando-se $\pm 0,5 \%$, durante os períodos de estabilização, e reduzindo-se essa tolerância para $\pm 0,2 \%$ no momento das medições. O conteúdo total de harmônicas da tensão de alimentação não deve exceder $3 \%$. Para o ensaio de vida, a tolerância é de $\pm 2 \%$ e o conteúdo total de harmônicas da tensão de alimentação também não pode exceder $3 \%$.

A alimentação das lâmpadas em ensaio no LABLUX é feita por meio de uma fonte de tensão controlada do fabricante Califórnia Instruments modelo 300lix, conforme se apresenta na Figura 1.

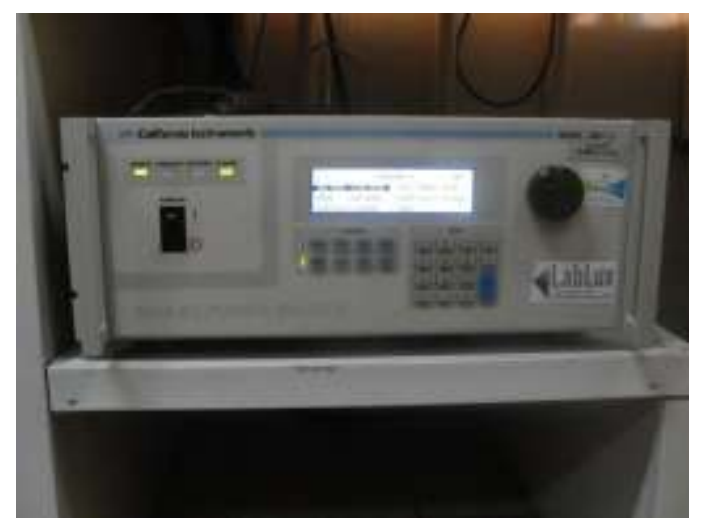

Figura 1. Fonte Controlada Califórnia Instruments

Efetua-se a medição de intensidade fluxo luminoso com o multifotômetro Everfine Photo-2000J ilustrado na Figura 2. Os ensaios são realizados em esfera integradora com sensor posicionado após anteparo em ponto determinado no projeto da esfera. A Figura 3 ilustra a esfera instalada no LABLUX utilizada neste estudo.

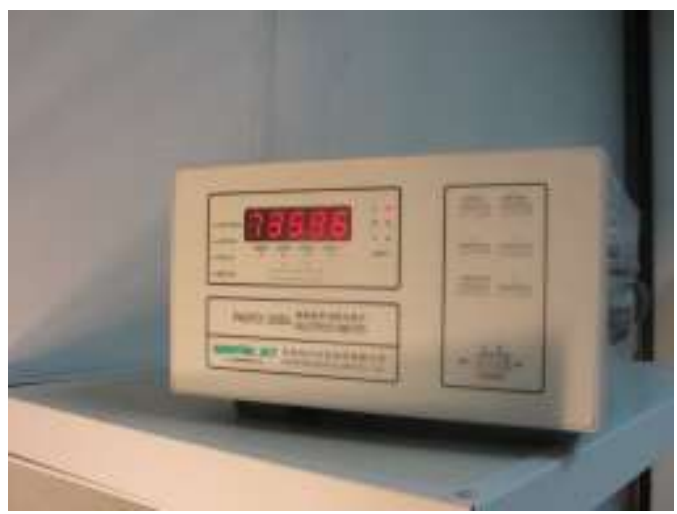

Figura 2. Multifotômetro 


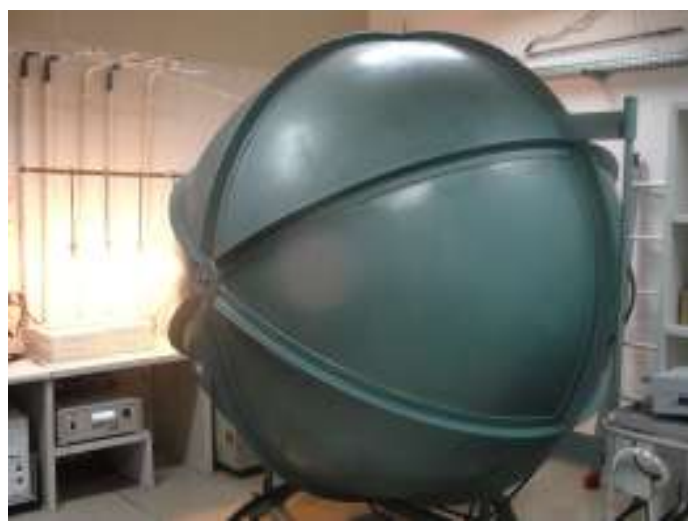

Figura 3. Esfera Integradora instalada no LABLUX

A qualidade de energia nos ensaios é avaliada por um analisador/watimetro Yokokawa WT-210, ilustrado na Figura 4.

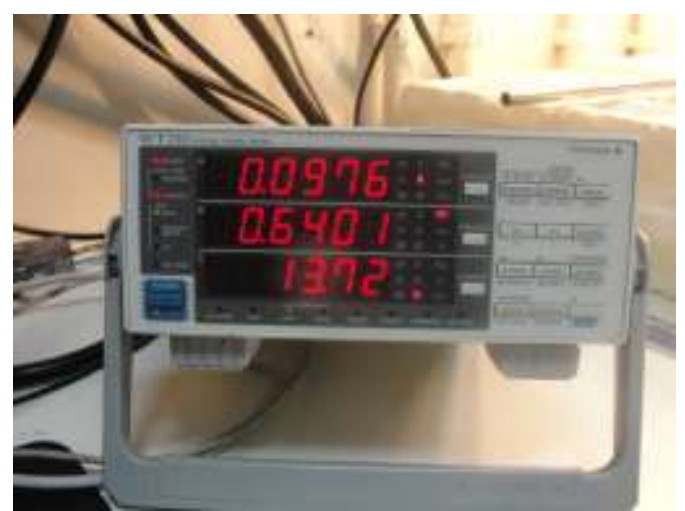

Figura 4. Analisador de Qualidade de Energia empregado

\section{DESCRICÃO DA METODOLOGIA DE MEDIÇẨO}

O PRODIST - Módulo 8 em sua revisão 4, aprovada na Agencia Nacional de Energia Elétrica - ANEEL pela Revisão Normativa $n^{\circ}$ 469/2011 (ANEEL, 2012), estabelece os procedimentos relativos à qualidade de energia elétrica - QEE, e sua abrangência atinge a todos os consumidores conectados a rede de distribuição.

Este trabalho visa avaliar o comportamento das LFC's frente aos limites de tensão estabelecidos em (ANEEL, 2012). No item 2.5.2.3 do Módulo 8 do PRODIST, apresentam-se as faixas de classificação de tensões - tensões em regime permanente. São transcritos na Tabela 1 todos os valores referentes à classe de tensão corresponde ao consumidor final no Brasil.
Tabela 1. Pontos de conexão em tensão igual ou inferior a $1 \mathrm{kV}(220 / 127 \mathrm{~V})$

\begin{tabular}{cc}
\hline $\begin{array}{c}\text { Tensão de } \\
\text { Atendimento (TA) }\end{array}$ & $\begin{array}{c}\text { Faixa de Variação da Tensão de } \\
\text { Leitura (TL) em Volts }\end{array}$ \\
\hline Adequada & $(201 \leq \mathrm{TL} \leq 231) /(116 \leq \mathrm{TL} \leq 133)$ \\
\hline Precária & $(189 \leq \mathrm{TL} \leq 201$ ou $231 \leq \mathrm{TL} \leq 233) /$ \\
& $(109 \leq \mathrm{TL} \leq 116$ ou $133 \leq \mathrm{TL} \leq 140)$ \\
\hline Critica & $(\mathrm{TL}<189$ ou $\mathrm{TL}>233) /(\mathrm{TL}<109$ \\
& ou TL $>140)$ \\
\hline
\end{tabular}

A metodologia consistiu em ensaiar e coletar dados para análise, verificando-se o comportamento das grandezas padronizadas para a LFC's, quando operando dentro dos limites de tensões 201 e $231 \mathrm{~V}$, para lâmpadas alimentadas em $220 \mathrm{~V}$ - que é o nível de tensão das amostras ensaiadas neste estudo, e 116 e $133 \mathrm{~V}$ para lâmpadas alimentadas em $110 \mathrm{~V}$. Os dados são coletados em 16 pontos para cada amostra, sendo os pontos de 1 a 5 em passos de $1 \%$ acima da tensão nominal até $+5 \%$, o ponto 6 na tensão nominal de referência, e os pontos 7 a 16, em passos de $1 \%$ abaixo da tensão nominal até $-10 \%$.

O $1^{\circ}$ indicador a ser analisado foi o fator de potência, avaliando-se $\mathrm{O}$ comportamento das LFC's com relação ao item 1.8 da portaria $n^{\circ} \quad 489 / 2010$ (INMETRO, 2010). No item fator de potencia deve-se considerar a distorção da forma de onda da corrente de alimentação, conforme Equação 1.

$$
F P=\frac{\cos \phi}{\sqrt{1+D H T^{2}}}
$$

onde : FP é o fator de potência

$\cos \phi$ é o fator de deslocamento

DHT é a Distorção Harmônica Total de Corrente

As lâmpadas fluorescentes compactas devem atender os valores indicados na Tabela 2 (INMETRO, 2010), com tolerância de $\pm 0,05$ entre o valor declarado pelo fabricante e o medido. Os ensaios realizados sob as condições limites do PRODIST avaliaram este parâmetro.

Tabela 2. Fator de potência mínimo

\begin{tabular}{cc}
\hline $\begin{array}{c}\text { Potência da Lâmpada } \\
(\mathbf{W})\end{array}$ & $\begin{array}{c}\text { Fator de Potência } \\
\text { Mínimo }\end{array}$ \\
\hline $\mathrm{P} \leq 25$ & 0,5 \\
\hline $\mathrm{P} \geq 25$ & 0,92 \\
\hline
\end{tabular}


$\mathrm{O} 2^{\circ}$ indicador estudado é a potência média medida. Os valores da potência devem estar entre os limites de 5\% a mais ou $10 \%$ a menos do que a potência declarada pelo fabricante na embalagem, seguindo o requisito de (INMETRO, 2010) para ensaio de concessão. Este estudo utiliza lâmpadas com potência de $15 \mathrm{~W}$ declarada em embalagem.

O $3^{\circ}$ indicador avaliado é o nível de Eficiência Energética determinado pela razão entre o fluxo luminoso médio medido e a potência média medida. A Tabela 3 apresenta de forma resumida os valores considerados como mínimos por (INMETRO, 2010).

Tabela 3. Níveis Mínimos de Eficiência

\begin{tabular}{cc}
\multicolumn{2}{c}{ Energética } \\
\hline LFC com invólucro & $\begin{array}{c}\text { Nível Mínimo } \\
\text { (lumens/Watt) }\end{array}$ \\
\hline Potência da Lâmpada $\leq 8 \mathrm{~W}$ & 40 \\
\hline $8 \mathrm{~W}<$ Potência da Lâmpada $\leq 15 \mathrm{~W}$ & 40 \\
\hline $15 \mathrm{~W}<$ Potência da Lâmpada $\leq 25 \mathrm{~W}$ & 44 \\
\hline 25 W < Potência da Lâmpada & 45 \\
\hline
\end{tabular}

Apresentam-se neste trabalho outros três indicadores para comparar respostas das lâmpadas ensaiadas entre os limites de tensão mínimo e máximo. $\mathrm{O} 4^{\circ}$ indicador é $\Delta I V$ que avalia a relação entre as variações de corrente consumidas frente à variação da tensão aplicada, conforme Equação 2.

$$
\Delta I V=\frac{\left(\frac{I_{n m}-I_{n 6}}{I_{n 6}}\right)}{\left(\frac{V_{n m}-V_{n 6}}{V_{n 6}}\right)} \cdot 100 \%
$$

onde: $I_{n m}$ - corrente, em $\mathrm{mA}$, da amostra $n$ para a medição $m$;

$I_{n 6}$ - corrente, em mA, da amostra $n$ para medição 6 , que corresponde ao ensaio com tensão nominal;

$V_{n m}$ - tensão, em $\mathrm{V}$, aplicada na amostra $n$ para a medição $m$; e

$V_{n 6}$ - tensão, em $\mathrm{V}$, aplicada na amostra $n$ para medição 6 , que corresponde ao ensaio com tensão nominal.

$\mathrm{O} 5^{\circ}$ indicador é o $\triangle F P V$ que analisa a relação entre as variações do fator de potência frente à variação da tensão aplicada, conforme Equação 3.

$$
\Delta F P V=\frac{\left(\frac{F P_{n m}-F P_{n 6}}{F P_{n 6}}\right)}{\left(\frac{V_{n m}-V_{n 6}}{V_{n 6}}\right)} .100 \%
$$

onde: $F P_{n m}$ - fator de potência da amostra $n$ para a medição $m$; e

$F P_{n 6}$ - fator de potência da amostra $n$ para medição 6 , que corresponde ao ensaio com tensão nominal

O $6^{\circ}$ indicador e ultimo indicador é o $\Delta F V$ que observa a relação entre variações do fluxo luminoso frente à variação da tensão aplicada, conforme Equação 4.

$$
\Delta F V=\frac{\left(\frac{F_{n m}-F_{n 6}}{F_{n 6}}\right)}{\left(\frac{V_{n m}-V_{n 6}}{V_{n 6}}\right)} \cdot 100 \%
$$

onde: $F_{n m}$ - fluxo luminoso, em lumens, da amostra $n$ para a medição $m$; e

$F_{n 6}$ - fluxo luminoso, em lumens, da amostra $n$ para medição 6 , que corresponde ao ensaio com tensão nominal.

\section{RESULTADOS DAS MEDIÇÕES}

Foram realizadas 16 medições em cada uma das 10 amostras. Para efeito de comparação, todas as LFC's testadas possuem a mesma potência nominal declarada pelos fabricantes em suas respectivas embalagens individuais $-15 \mathrm{~W}$, tensão de alimentação $220 \mathrm{~V}$ e modelo de rosca E-27, e foram aprovadas em testes anteriores para comercialização no mercado brasileiro.

Apresentam-se a seguir em forma de Tabelas os resultados obtidos para os ciclos de testes.

A Tabela 4 apresenta os resultados para o fator de potência. Relacionam-se os menores e maiores valores encontrados em cada uma das amostras. Todos os menores valores de fator de potência foram obtidos na condição de $220 \mathrm{~V}+5 \%$ e os maiores valores na condição $220 \mathrm{~V}+10 \%$. Cabe 
lembrar que o valor mínimo referenciado na regulamentação é de 0,5 com variação de $\pm 0,05$ na tensão nominal, conforme apresentado na Tabela 2. Estão também indicados na Tabela 4 às distorções harmônicas totais de corrente encontradas na respectiva medição indicada.

A Figura 5 ilustra um exemplo de espectro harmônico de corrente na amostra 2 quando submetida à tensão $220 \mathrm{~V}-10 \%$.

Avaliando-se o $2^{\circ}$ indicador deste estudo - Potência Média - apresentam-se na Tabela 5, os valores máximos e mínimos medidos em cada amostra. Sendo as amostras todas de $15 \mathrm{~W}$, os valores encontrados possuem limites regulamentados de $15,75 \mathrm{~W}$ e $13,5 \mathrm{~W}$. Os maiores valores encontrados ocorreram todos no ensaio com $220 \mathrm{~V}+5 \%$ e os menores valores no ensaio $220 \mathrm{~V}-10 \%$.

Tabela 4. Resultados para o indicador fator de potência considerando a correção para distorções harmônicas

\begin{tabular}{ccc}
\hline $\begin{array}{c}\mathbf{N}^{\mathbf{0}} \mathbf{d a} \\
\text { Amostra }\end{array}$ & $\begin{array}{c}\text { Menor fator de } \\
\text { potência medido não } \\
\text { corrigido e THD \% na } \\
\text { medição }\end{array}$ & $\begin{array}{c}\text { Maior fator de } \\
\text { potência medido não } \\
\text { corrigido e THD \% } \\
\text { na medição }\end{array}$ \\
\hline 1 & $0,6362-101,2$ & $0,6477-93,6$ \\
\hline 2 & $0,6346-101,8$ & $0,6459-94,91$ \\
\hline 3 & $0,6341-102,1$ & $0,6451-95,11$ \\
\hline 4 & $0,6383-99,9$ & $0,6507-92,1$ \\
\hline 5 & $0,6380-99,98$ & $0,6498-92,33$ \\
\hline 6 & $0,6342-102,1$ & $0,6452-94,88$ \\
\hline 7 & $0,6359-101,3$ & $0,6474-94,2$ \\
\hline 8 & $0,6362-100,9$ & $0,6478-93,72$ \\
\hline 9 & $0,6344-102,2$ & $0,6462-94,9$ \\
\hline 10 & $0,6339-102,4$ & $0,6459-94,6$ \\
\hline
\end{tabular}

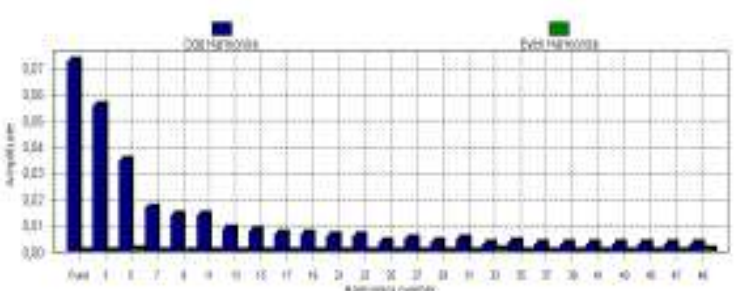

Figura 5. Espectro Harmônico de Corrente na Amostra 2 submetida à tensão $220 \mathrm{~V}$ $10 \%$

$\mathrm{O} \quad 4^{\circ}$ indicador avalia as variações percentuais de corrente frente às variações de tensão. Não existem valores recomendados ou padronizados, mas introduz-se este indicador para apoiar as discussões com respeito a desempenho das LFC's. A Tabela 7 apresenta o quadro resumo com valores máximos e mínimos encontrados, com suas respectivas amostras identificadas.

Tabela 5. Resultados para o indicador Potência Média

\begin{tabular}{ccc}
\hline $\begin{array}{c}\mathbf{N}^{\mathbf{0}} \text { da } \\
\text { Amostra }\end{array}$ & $\begin{array}{c}\text { Maior potência } \\
\text { medida }\end{array}$ & $\begin{array}{c}\text { Menor potência } \\
\text { medida }\end{array}$ \\
\hline 1 & 14,3 & 12,5 \\
\hline 2 & 14,26 & 12,54 \\
\hline 3 & 14 & 12,21 \\
\hline 4 & 14,9 & 13,2 \\
\hline 5 & 14,29 & 12,77 \\
\hline 6 & 14,04 & 12,37 \\
\hline 7 & 14,5 & 12,7 \\
\hline 8 & 14,42 & 12,69 \\
\hline 9 & 13,95 & 12,15 \\
\hline 10 & 13,9 & 12,3 \\
\hline
\end{tabular}

O $3^{\circ}$ indicador, nível de eficiência energética, que deve ser no mínimo 40 lumens/Watt para as amostras referenciadas neste estudo, tem os resultados mínimos e máximos encontrados na Tabela 6 , com a indicação da variação percentual no teste registrado.

Tabela 6. Resultados para o indicador Eficiência Energética

\begin{tabular}{ccc}
\hline $\begin{array}{c}\mathbf{N}^{0} \text { da } \\
\text { Amostra }\end{array}$ & $\begin{array}{c}\text { Maior Eficiência } \\
\text { Energética / \% Tensão }\end{array}$ & $\begin{array}{c}\text { Menor Eficiência } \\
\text { Energética / \% Tensão }\end{array}$ \\
\hline 1 & $54 /-10$ & $52,5 /+5$ \\
\hline 2 & $55,1 /-10$ & $54,1 /+5$ \\
\hline 3 & $64,1 /-4$ & $63,5 /+5$ \\
\hline 4 & $48,8 / 0$ & $46,6 /-10$ \\
\hline 5 & $51,2 /+10$ & $50,2 /+5$ \\
\hline 6 & $56,7 /-5$ & $55,4 /+5$ \\
\hline 7 & $53,6 /-10$ & $52,5 /+4$ \\
\hline 8 & $53,7 /-10$ & $52,1 /+5$ \\
\hline 9 & $57,7 /-10$ & $55,1 /+5$ \\
\hline 10 & $65,2 /-3$ & $64 /+5$ \\
\hline
\end{tabular}

O $5^{\circ}$ indicador avalia as variações percentuais do fator de potência frente às variações de tensão. Não existem valores recomendados ou padronizados, mas introduz-se este indicador, como no caso anterior, para apoiar as discussões com respeito ao desempenho das LFC's. A Tabela 8 apresenta o quadro resumo com valores máximos e mínimos encontrados, com suas respectivas amostras identificadas. 
Tabela 7. Resultados para o indicador $\Delta I V$

\begin{tabular}{ccc}
\hline $\begin{array}{c}\mathbf{N}^{0} \text { da } \\
\text { Amostra }\end{array}$ & $\begin{array}{c}\text { Maior variação } \\
\text { percentual / \% Tensão }\end{array}$ & $\begin{array}{c}\text { Menor variação } \\
\text { percentual / \% Tensão }\end{array}$ \\
\hline 1 & $36,15 /+2$ & $2,06 /+5$ \\
\hline 2 & $30,86 /+1$ & $7,71 /+4$ \\
\hline 3 & $-20,92 /+1$ & $0 /-3$ \\
\hline 4 & $59,17 /+1$ & $0 /+2$ \\
\hline 5 & $-30,15 /-1$ & $0 /+4$ \\
\hline 6 & $-20,85 /-1$ & $0 /+3$ \\
\hline 7 & $10,14 /-1$ & $0 /+1$ \\
\hline 8 & $10,19 /-1$ & $2,03 /+5$ \\
\hline 9 & $26,34 /+2$ & $0 /-5$ \\
\hline 10 & $-1578 /-4$ & $0 /+5$ \\
\hline
\end{tabular}

O $6^{\circ}$ indicador deste estudo avalia as variações percentuais do fluxo luminoso frente as variações de tensão. A Tabela 9 apresenta o quadro resumo com valores máximos e mínimos percentuais encontrados e a identificação da amostra.

Tabela 8. Resultados para o indicador $\triangle F P V$

\begin{tabular}{ccc}
\hline $\begin{array}{c}\mathbf{N}^{0} \text { da } \\
\text { Amostra }\end{array}$ & $\begin{array}{c}\text { Maior variação } \\
\text { percentual / \% } \\
\text { Tensão }\end{array}$ & $\begin{array}{c}\text { Menor variação } \\
\text { percentual / \% } \\
\text { Tensão }\end{array}$ \\
\hline 1 & $-12,15 /-9$ & $-6,25 /+1$ \\
\hline 2 & $-15,67 /-1$ & $-6,27 /+1$ \\
\hline 3 & $-26,67 /+2$ & $-14,11 /+1$ \\
\hline 4 & $-29,68 /-2$ & $-1,04 /+3$ \\
\hline 5 & $-30,51 /-1$ & $0,0 /+4$ \\
\hline 6 & $-14,11 /+1$ & $-9,41 /-2$ \\
\hline 7 & $-17,18 /+1$ & $--3,12 /-1$ \\
\hline 8 & $-40,76 /-1$ & $-1,04 /+3$ \\
\hline 9 & $-18,82 /+1$ & $-7,84 /+2$ \\
\hline 10 & $-50,00 /+1$ & $0,52 /-3$ \\
\hline
\end{tabular}

Tabela 9. Resultados para o indicador $\Delta F V$

\begin{tabular}{ccc}
\hline $\begin{array}{c}\mathbf{N}^{\mathbf{0}} \text { da } \\
\text { Amostra }\end{array}$ & $\begin{array}{c}\text { Maior variação } \\
\text { percentual / \% Tensão }\end{array}$ & $\begin{array}{c}\text { Menor variação } \\
\text { percentual / \% Tensão }\end{array}$ \\
\hline 1 & $147,1 /-1$ & $49,66 /-7$ \\
\hline 2 & $109,34 /+1$ & $27,7 /-1$ \\
\hline 3 & $90,9 /-9$ & $61,9 /+1$ \\
\hline 4 & $214,2 /-1$ & $24,87 /+3$ \\
\hline 5 & $117,51 /+1$ & $27,15 /+5$ \\
\hline 6 & $77,73 /+3$ & $55,43 /+5$ \\
\hline 7 & $86,09 /+1$ & $56,57 /-2$ \\
\hline 8 & $69,02 /-10$ & $37,02 /+1$ \\
\hline 9 & $78,36 /+2$ & $51,72 /+5$ \\
\hline 10 & $77,19 /-10$ & $59,3 /+5$ \\
\hline
\end{tabular}

\section{CONCLUSÕES}

Este estudo objetivou conhecer um pouco mais do comportamento das LFC's disponíveis no mercado frente aos limites de tensão permitidos aos sistemas de distribuição no ponto de conexão ao consumidor. Escolheram-se 10 amostras de fabricantes diferentes disponíveis no mercado varejista em diversas cidades da região sudeste do Brasil, e que previamente possuíam selo INMETRO, caracterizandoas como aprovadas anteriormente por laboratório autorizado. Estas amostras possuem mesmas características elétricas. Efetuaram-se diversas medições em laboratório acreditado pelo INMETRO para avaliações energéticas e de luminotécnica.

Baseando-se os testes na prática voltada à aprovação de lâmpadas para o mercado em condições nominais e com equipamentos certificados e rastreados, ensaiaram-se as amostras em 16 níveis de tensão diferentes e compararam-se os resultados para três indicadores típicos normatizados e outros três propostos pelos autores do artigo para identificar algumas características nos ensaios.

Com relação ao aspecto fator de potência, percebe-se que todas as lâmpadas possuem valor acima do normatizado mesmo quando sujeitas as variações de tensões limites do PRODIST. Este valor apesar de estar acima do limite regulamente é considerado baixo considerando os aspectos de perdas e é comprovado que este valor é impactado diretamente pelo alto conteúdo harmônico característicos das lâmpadas LFC's.

No aspecto potência medida, as amostras apresentaram valores sempre inferiores ao nominal declarado pelo fabricante, mas quando aplicados os limites de tensão de rede, todas as amostras possuem pelo menos uma leitura abaixo do limite inferior recomendado. Quando se pensa em consumo, este valor pode induzir a avaliação de que as unidades LFC estão ótimas, mas quando, observa-se o aspecto variação do fluxo luminoso $\Delta F V$ as variações percentuais são elevadas, podendo-se caracterizar por não proporcionalidade do fluxo frente a variações de tensão.

Analisando-se os indicadores de variação de corrente e variação do fator de potência frente a variações de tensão, observa-se que não existe uma uniformidade entre as amostras com relação a limites e com relação a percentual 
de tensão que proporciona maior variação. Entende-se que esta não uniformidade caracteriza que os projetos das LFC ainda estão em desenvolvimento e que uma solução única e energeticamente mais eficiente ainda não foi encontrada.

Em uma comparação entre as 10 amostras de mesma potência e tensão disponíveis no mercado, pode-se dizer que as variações do fator de potência entre as diversas amostras é praticamente nula, podendo-se considerar que todas possuem o mesmo comportamento com relação a este parâmetro quando submetidas a variações de tensões limites. Com relação à distorção harmônica total de corrente, todas as amostras possuem valores superiores a $90 \%$, o que impacta diretamente no fator de potência final da lâmpada. Com relação a este parâmetro, os fabricantes podem trabalhar melhor seus projetos com o objetivo de reduzir este percentual e consequentemente melhorar $\mathrm{o}$ fator de potência final da LFC.

Comparando-se as amostras no aspecto eficiência energética, percebe-se uma grande variação entre os fabricantes. Mediu-se uma eficiência energética de 46,6 $\mathrm{lm} / \mathrm{W}$ para a amostra 4 e $64,1 \mathrm{~lm} / \mathrm{W}$ para a amostra 3. Tomando-se como referência o valor de $40 \mathrm{~lm} / \mathrm{W}$, todas as amostras atenderam ao requisito normatizado, mesmo sob influência de variação de tensão, mas a diferença percentual entre o valor máximo encontrado e a referência chega a 60,25\%, que chama a atenção, caracterizando uma não uniformidade tecnológica dos fabricantes.

Ressalta-se que a análise de eficiência energética é o parâmetro que contempla os resultados obtidos com as medições de fluxo luminoso, visto que este parâmetro possui relação direta com a potência consumida da rede.

Mesmo estando todas as amostras de acordo com a norma vigente, o que possibilita uma segurança ao consumidor brasileiro quando da aquisição de uma LFC no mercado varejo, percebe-se com este artigo que dois novos pontos devem ser discutidos em futuros projetos: desenvolvimento de LFC's que atendam a todos os parâmetros dentro das tensões limites de distribuição que alimentam os consumidores finais e apresentar novos indicadores a serem desenvolvidos na avaliação da qualidade dos produtos ofertados ao mercado de iluminação, incluindo estes novos parâmetros na qualificação de eficiência do produto para o mercado.

\section{REFERÊNCIAS}

ANEEL. Procedimentos de Distribuição de Energia Elétrica no Sistema Elétrico Nacional - PRODIST- Módulo 8. Disponível em: <http:www.aneel.gov. BR/arquivos/PDF/módulo8_Revisão_4.pdf >. Acesso em 04 out 2012

BLANCO, A.M.; PARRA,E.; SCHAU,H. Comparison between compact fluorescent lamps distributed in Colombia and Germany. In: $46^{\text {th }}$ International Universities Power Engineering Conference - UPEC 2011, p.1-5, 2011.

COCA,E.; POPA,V.; BUTA,G. Compact Fluorescent Lamps Electromagnetic Compatibility Measurements and Performance Evaluation. In: International Conference on Computer as a Tool EUROCON 2011, p.1-4, 2011.

DILAI,L.; LANXIANG,Z.; ZISSIS,G.; ZHIGUO,L. Investigation of Electrical Parameters in Compact Fluorescent Lamps. In: Conference Record of the 2005 Industry Applications Conference - $40^{\text {th }}$ IAS Annual Meeting, p.2335-2339, 2005.

INMETRO. Portaria $n^{\circ} 489$ de 08/12/2010. Disponível em: < http: www.aladi.org/nsfaladi/normasTecnicas. nsf/09267198f1324b6403274960062343c/e 380ed588b45568f032579e4004f416e/e380 ed588b45568f /\$FILE/Portaria\% 20N\%C2\%B0\%20489-2010.pdf>. Acesso em 04 out 2012.

MAY, C.; COLLINS,E.R. An Investigation of the response of incandescent lamp and compact fluorescent lamps to voltage fluctuations. In: $14^{\text {th }}$ International Conference on Harmonics a Quality of Power-ICHQP 2010, p.1-8, 2010. 
PRESIDÊNCIA DA REPÚBLICA. Decreto $n^{\circ} 10.295$ de 17/10/2001. Disponível em: <http:planalto.gov.br/ ccivil03/decreto/2001/D4059.htm>. Acesso em 05 jan 2013.

PRESIDÊNCIA DA REPÚBLICA. Decreto $n^{\circ} 4.059$ de 19/04/2001. Disponível em: <http:planalto.gov.br/ ccivil_03/Leis/LEIS_2001/L10295.htm>Ac esso em 05 jan 2013.

PROCEL ELETROBRÁS. Apresentação. Disponível em: <http:www.eletrobras. gov.br/elb/procel/main.asp?TeamID=\% 7B6 7469FA5-276E-431F-B9C0-4063049 8F40630498EE\%7D>Acesso em 05 jan 2013.

SHAFI,M.A. McMAHON,R.A. Influence of voltage and frequency dimming on power losses in HF Electronic Ballasts for Compact Fluorescent Lamps. In: Conference Record of the 2006 Industry Applications Conference $-41^{\text {th }}$ IAS Annual Meeting, p.2193-2197, 2006.

SCHINKELSHOEK,M.; WATSON,N.R. HEFFERNAN,B. The characteristics of CFL's beyond the harmonics. In: $20^{\text {th }}$ Australasian Universities Power Engineering Conference - AUPEC 2010, p.1-6, 2010.

WATSON, N.R.; SCOTT,T.L.; HIRSCH, S.J.J. Implications for Distribution Networks of High Penetration of Compact Fluorescent Lamps. IEEE Transactions on Power Delivery, v.24, p.1521-1528, 2009. 